\title{
Dispositivos inhaladores en la EPOC: ¿Cuál utilizar?
}

Which inhaler device in COPD?

Francisco López García, Cristina Bas Reina

Servicio de Medicina Interna. Hospital General de Elche, Alicante

\section{Introducción}

El tratamiento de la EPOC ha experimentado un avance muy notable en los últimos años debido a la aparición de nuevos broncodilatadores. Actualmente se dispone de broncodilatadores y corticoides inhalados que se pueden administrar una sola vez al día y cada 12 horas, con lo que las combinaciones pueden ser múltiples (Tabla 1). Por tanto, somos capaces de individualizar el tratamiento de los pacientes con EPOC de la misma forma que se hace en otras enfermedades crónicas, como la hipertensión arterial o la diabetes mellitus ${ }^{1-3}$.

Por otra parte, la EPOC, como el asma, tiene la particularidad que la vía de administración de los fármacos se realiza por vía inhalada. En este sentido, la vía inhalada en comparación con la vía oral presenta ventajas evidentes: se usan dosis menores, el inicio de acción es más rápido y hay menos

Tabla 1. Tratamiento farmacológico con broncodilatadores y corticoides inhalados en la EPOC

\begin{tabular}{|c|c|c|}
\hline $\begin{array}{l}\text { Grupo } \\
\text { farmacológico }\end{array}$ & $\begin{array}{l}\text { Tiempo de } \\
\text { acción }\end{array}$ & Principio activo \\
\hline \multirow{3}{*}{ Beta-2-agonistas } & Corta (SABA) & $\begin{array}{l}\text { Salbutamol } \\
\text { Terbutalina }\end{array}$ \\
\hline & Larga (LABA) & $\begin{array}{c}\text { Salmeterol } \\
\text { Formoterol } \\
\text { Indacaterol } \\
\text { Vilanterol } \\
\text { Olodaterol }\end{array}$ \\
\hline & $\mathrm{LABA}+\mathrm{Cl}$ & $\begin{array}{c}\text { Salmeterol + Fluticasona } \\
\text { Formoterol + Budesonida } \\
\text { Formoterol + Beclometasona } \\
\text { Vilanterol + Fluticasona }\end{array}$ \\
\hline \multirow{3}{*}{ Anticolinérgicos } & Corta (SAMA) & Bromuro de ipratropio \\
\hline & Larga (LAMA) & $\begin{array}{l}\text { Bromuro de tiotropio } \\
\text { Bromuro de aclidinio } \\
\text { Bromuro de glicopirronio } \\
\text { Bromuro de umeclidinio }\end{array}$ \\
\hline & LABA + LAMA & $\begin{array}{c}\text { Indacaterol + glicopirronio } \\
\text { Vilanterol + umeclidinio } \\
\text { Formoterol + aclidinio* } \\
\text { Olodaterol + Tiotropio* }\end{array}$ \\
\hline
\end{tabular}

Siglas en inglés : SABA: beta-dos de acción corta. SAMA: anticolinérgico de acción corta. LAMA: anticolinérgico de acción larga. LABA: beta-dos de acción larga. Cl: corticoide inhalado.

*Nuevas combinaciones LAMA-LABA actualmente no comercializados en España. distribución sistémica del fármaco con lo que disminuyen los efectos adversos. Sin embargo, la gran desventaja es que se necesitan aparatos (dispositivos) para administrar el fármaco y la técnica de su uso es compleja ${ }^{4}$. Actualmente existe una amplia gama de nuevos dispositivos a lo que se añade la modificación y perfeccionamiento de los ya existentes, lo que incorpora un nuevo grado de dificultad en el manejo global del paciente con EPOC ${ }^{4,5}$ (Tabla 2). Es bien conocido que no se va a producir el control de la enfermedad y va a ser inevitable el fracaso terapéutico si el uso del dispositivo y la técnica inhalatoria no son los adecuados ${ }^{6}$. Todo esto obliga a los profesionales que atienden a los pacientes con EPOC a enseñar y adiestrar perfectamente al enfermo en la buena utilización del dispositivo y en que realice la técnica inhalatoria de forma correcta.

Tabla 2. Dispositivos de inhalación más comunes en la EPOC

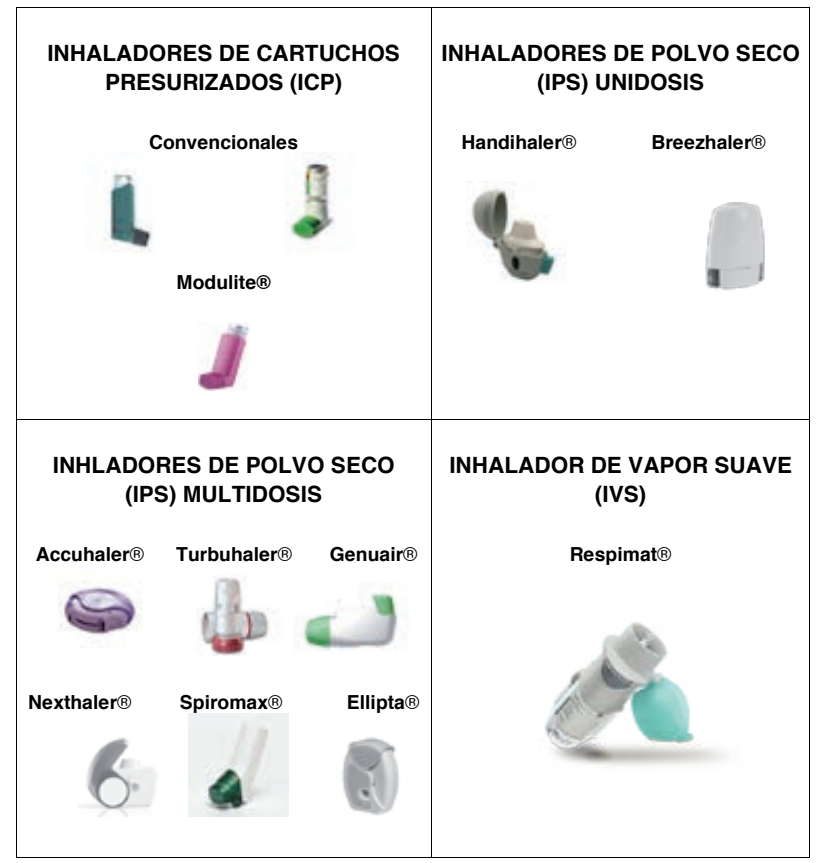

\section{Dispositivos y cámaras de inhalación: Ventajas e inconvenientes}

Los dispositivos de inhalación que se usan en la EPOC se clasifican en los inhaladores de cartucho presurizado (ICP), los dispositivos de polvo seco (IPS), y el inhalador de vapor suave (IVS) ${ }^{7-9}$. En la tabla 3 están resumidas las ventajas e inconvenientes de cada uno de ellos. 
Tabla 3. Dispositivos de inhalación en la EPOC. Ventajas e inconvenientes

\begin{tabular}{|c|c|c|c|c|}
\hline \multicolumn{3}{|l|}{ Dispositivos } & Ventajas & Inconvenientes \\
\hline \multirow[t]{2}{*}{$\begin{array}{l}\text { Inhaladores } \\
\text { de cartuchos } \\
\text { presurizados } \\
\text { (ICP) }\end{array}$} & \multicolumn{2}{|c|}{$\begin{array}{l}\text { Dispositivos inhaladores } \\
\text { presurizados de dosis } \\
\text { medida convencionales }\end{array}$} & $\begin{array}{l}\text { - No requiere energía } \\
\text { externa. } \\
\text { - Rapidez en el uso } \\
\text { - Percepción } \\
\text { inhalación. } \\
\text { - Precisa flujos bajos. } \\
\text { - Dosis liberada } \\
\text { independiente de la } \\
\text { maniobra. } \\
\text { - Útil en VM. } \\
\text { - Económico. } \\
\end{array}$ & $\begin{array}{l}\text { - Difícil coordinación. } \\
\text { - Alto depósito } \\
\text { orofaríngeo, } \\
\text { - Bajo depósito } \\
\text { pulmonar. } \\
\text { - Ausencia contador de } \\
\text { dosis. } \\
\text { - Requiere agitación } \\
\text { antes de usar. }\end{array}$ \\
\hline & \multicolumn{2}{|c|}{$\begin{array}{l}\text { Dispositivos inhaladores } \\
\text { presurizados con dosis } \\
\text { medida de partículas } \\
\text { extrafinas (Modulite@) }\end{array}$} & $\begin{array}{l}\text { - Mayor depósito } \\
\text { pulmonar. } \\
\text { - Facilita } \\
\text { coordinación. } \\
\text { - Flujos inspiratorios } \\
\text { bajos. } \\
\text { - Contador de dosis. }\end{array}$ & $\begin{array}{l}\text { - Ausencia contador de } \\
\text { dosis. } \\
\text { - Refrigeración durante } \\
\text { almacenamiento. } \\
\text { - } 3 \text { meses de } \\
\text { caducidad. }\end{array}$ \\
\hline \multirow[b]{2}{*}{$\begin{array}{l}\text { Dispositivos } \\
\text { de polvo seco } \\
\text { (IPS) }\end{array}$} & Unidosis & $\begin{array}{l}\text { Breezhaler } 尺 \\
\text { Handihaler } ®\end{array}$ & \multirow{2}{*}{$\begin{array}{l}\text { - Eficacia clínica igual } \\
\text { o superior a ICP. } \\
\text { - Fácil coordinación } \\
\text { pulsación- } \\
\text { inhalación. } \\
\text { - Indicador de dosis. } \\
\text { - Pequeños, fácil } \\
\text { manejo y discretos. } \\
\text { - Suelen ser } \\
\text { preferidos por } \\
\text { los pacientes y } \\
\text { sanitarios. }\end{array}$} & \multirow{2}{*}{$\begin{array}{l}\text { - Precisan inspiración } \\
\text { voluntaria y flujos } \\
\text { más altos que ICP. } \\
\text { - Mayor impacto } \\
\text { orofaríngeo. } \\
\text { - En algunos, si se } \\
\text { sopla se pierde la } \\
\text { dosis. } \\
\text { - En algún caso, no se } \\
\text { percibe la inhalación. } \\
\text { - Si se humedecen } \\
\text { pueden ser } \\
\text { ineficaces } \\
\text { - Son más caros que } \\
\text { los ICP. } \\
\text { - No útiles en VM. }\end{array}$} \\
\hline & Multidosis & 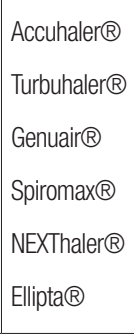 & & \\
\hline $\begin{array}{l}\text { Inhalador de } \\
\text { vapor suave } \\
\text { (IVS) }\end{array}$ & \multicolumn{2}{|l|}{ Respimat $\circledast$} & $\begin{array}{l}\text { - Bajo flujo } \\
\text { inspiratorio } \\
\text { - Elevado depósito } \\
\text { pulmonar } \\
\text { - Puede acoplarse a } \\
\text { cámara }\end{array}$ & \\
\hline
\end{tabular}

VM: ventilación mecánica

Los ICP son los primeros dispositivos que se utilizaron y siguen siendo los más prescritos en la EPOC. Se distingue el clásico o convencional, que es el más usado, y el sistema Modulite $®$, sólo disponible para formoterol y beclometasona. Los ICP son dispositivos manejables, fáciles de usar, de cómodo transporte y económicos. Su principal ventaja es que precisan de bajos flujos inspiratorios (<20-30 litros/minuto) para realizar la inhalación. Sin embargo, tienen claras desventajas en relación con Ios IPS como es la detención brusca de la inhalación (efecto frío-freón), el elevado depósito orofaríngeo, el bajo depósito pulmonar y sobre todo la necesidad de realizar una perfecta coordinación entre la pulsación y la inspiración profunda (maniobra fundamental en la técnica inhalatoria de los ICP). En el sistema Modulite $®$ los fármacos se encuentran en solución dentro del cartucho liberándose en partículas extrafinas, con lo que el aerosol formado es más lento favoreciendo un mayor depósito pulmonar y menor depósito orofarínge $0^{4,8}$.

Las cámaras de inhalación y espaciadores son recipientes que, acoplados a los ICP, permiten realizar la inhalación sin la necesidad de sincronizar la salida del fármaco con la inspiración. Las partículas pequeñas son las eficaces terapéuticamente y permanecen varios segundos en suspensión antes de ser inhaladas mientras que las partículas grandes, que son las inactivas, quedan adheridas en la cámara del espaciador. La consecuencia es que disminuye el depósito orofaríngeo de los fármacos con lo que aumenta el depósito en los pulmones. Por tanto, el uso acoplado de los ICP a las cámaras espaciadoras, aumenta claramente la eficacia de los primeros ${ }^{4,5}$.

Los IPS son dispositivos más modernos que los ICP y los preferidos por los pacientes y los profesionales que los atienden ${ }^{10}$. Son pequeños, portátiles y no contienen propelentes, pero la principal ventaja sobre los ICP es que se activan con la inspiración, por lo que se soluciona el problema de la necesidad de coordinar la activación del dispositivo y la inhalación, aspecto técnico fundamental en los ICP. Por tanto, la maniobra inhalatoria de los IPS es más sencilla, al no ser necesario coordinar inspiración con pulsación. El depósito pulmonar de los IPS es también mayor que el de los ICP. La principal desventaja es que precisa flujos inspiratorios mayores (30-90 litros/minuto) que los ICP y son también más caros $^{4,8}$. Existen dos tipos de IPS, los unidosis y multidosis. En el primer caso, los fármacos están en cápsulas con una única dosis y al accionar el dispositivo se perforan. Necesitan flujos inspiratorios altos y por tanto una maniobra inspiratoria profunda para realizar la correcta inhalación $n^{4,8,9}$. En EPOC las principales formas comercializadas de Ios IPS unidosis son el Handihaler® (tiotropio) y Breezhaler® (indacaterol, glicopirronio y la combinación de ambos). Los sistemas multidosis tienen el principio activo en un reservorio o bien se encuentran precargados en el propio dispositivo. En EPOC los principales dispositivos IPS multidosis son el Accuhaler ${ }^{\circledR}$ (salmeterol, fluticasona y la combinación), Turbuhaler $®$ (formoterol, budesonida y la combinación), Genuairß (aclidinio), NEXThaler $®$ (formoterol y beclometasona en combinación), Ellipta $®$ (fluticasona y vilanterol en asociación, umeclidinio y vilanterol en combinación) y Spiromax® (formoterol y budesonida en combinación) (Tabla 2).

El inhalador de vapor suave (IVS), también llamado de niebla fina, comparte características de los ICP y nebulizadores. Contiene una solución acuosa que genera una fina nube de aerosol que sale a una velocidad baja. Combina un depósito pulmonar muy alto, comparable a los IPS, pero con la ventaja de precisar un flujo inspiratorio también bajo, como sucede con los ICP. Además, puede acoplarse perfectamente a las cámaras de inhalación, con lo que su eficacia puede mejorar aún más ${ }^{4,5}$.

El primer y principal paso en el buen uso de los ICP es la adecuada sincronización entre la pulsación y la inhalación. En el caso de los ICP es el propio dispositivo el que realiza la fuerza que propulsa el fármaco. Por tanto, la inspiración debe ser lenta (4-5 segundos) y profunda para lograr un buen depósito bronquial. Si la inhalación se hace de manera rápida y enérgica, el fármaco se depositará a nivel orofaríngeo y no llegará a los pulmones. La técnica es radicalmente diferente con el uso de los IPS; el principal paso consiste en realizar 
una inspiración rápida y enérgica, ya que es el paciente el que hace la fuerza que propulsa el fármaco. Si se hace la inhalación de forma lenta, el polvo seco quedará sobre todo en el dispositivo y no llegará a los pulmones ${ }^{4,8}$.

\section{Criterios de elección \\ de los dispositivos inhaladores}

La elección del dispositivo adecuado es una tarea difícil y es fácil cometer errores. El mismo dispositivo le puede valer a un paciente individual y no servir a otro, debido a las características del dispositivo o a la peculiaridad de la técnica inhalatoria ${ }^{4,8,9}$. En líneas generales, para la elección inicial del dispositivo se debe de responder a dos cuestiones fundamentales:

1. ¿El paciente es capaz de coordinar de forma adecuada la inhalación con la activación del dispositivo?

2. ¿El paciente puede realizar una inhalación adecuada, definida como un flujo inspiratorio $>30 \mathrm{l} / \mathrm{min}$ ?

Con la respuesta positiva o negativa a ambas preguntas se establecen varias combinaciones de las que van a derivarse los posibles dispositivos a utilizar (Figura 1) ${ }^{4,7}$. Después de la elección inicial del dispositivo conviene tener en cuenta otros aspectos prácticos que influirán en la decisión del dispositivo a elegir:

1. En los IPS la fuerza propulsora proviene de la capacidad inhalatoria del enfermo. Por tanto, no se deben de utilizar en las crisis de broncoespasmo, en niños menores de 5 años y en pacientes inconscientes o con demencia avanzada.

2. Los IPS son los dispositivos de elección en los pacientes jóvenes, con vida activa y con buen nivel cognitivo. Sin embargo, por su complejidad en el manejo, los IPS sobre todo unidosis no son los electivos en los pacientes con deformidades, artritis, temblor esencial, enfermedad de Parkinson sintomática o con problemas visuales.

3. Es razonable que el paciente utilize el menor número de inhaladores posibles. Además, las pautas deben de ser simples y procurar que use dispositivos del mismo manejo y similar técnica inhalatoria. Como ejemplo, un paciente con EPOC puede estar en tratamiento con un ICP de rescate y tener asociados otros dispositivos como un IPS unidosis y otro IPS multidosis. En este caso, los dispositivos son diferentes y la técnica de inhalación también, pues, recordemos, en los ICP la inhalación tiene que ser lenta y progresiva y en los IPS rápida y enérgica. Para evitar estos casos que aumentan la probabilidad de errores, debe de evitarse pautas complejas, intentar combinar las mismas familias de dispositivos con similar técnica inhalatoria y reducir el número de dosis diarias.

4. La adaptación adecuada debe de hacerse en la dirección del dispositivo al paciente y no al revés. Debe de valorarse cada enfermo de forma individual y tener en cuenta
Figura 1. Algoritmo en la elección de los dispositivos inhaladores (fuente: referencia 4)

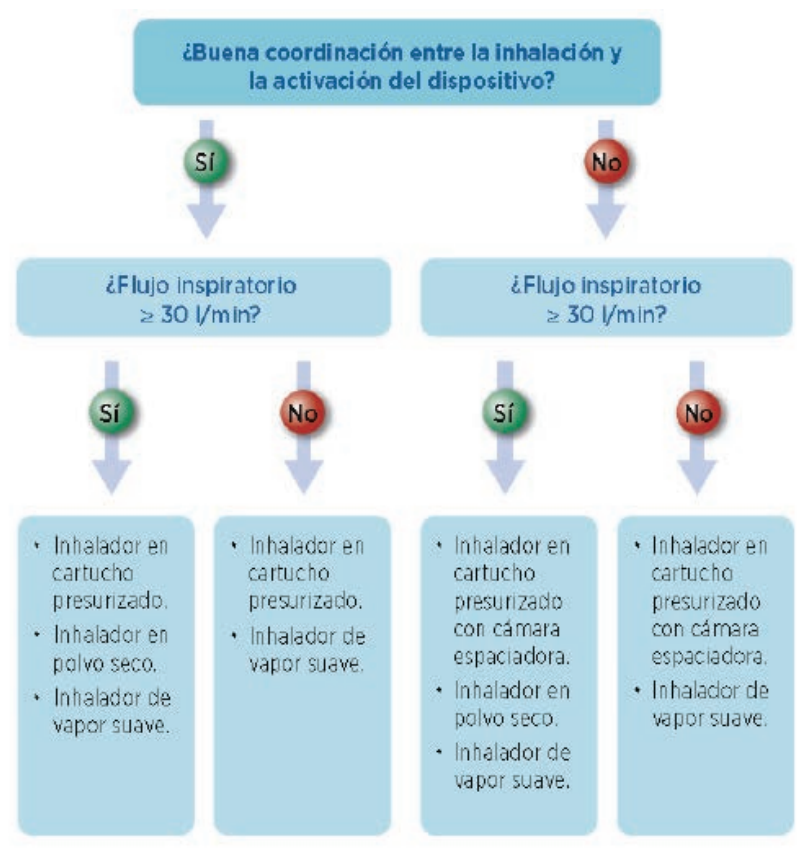

la gravedad de la EPOC que padece, las comorbilidades asociadas y sus habilidades físicas y psíquicas ${ }^{4,5,7-9}$.

El consenso SEPAR-ALAT sobre terapia inhalada del año 2013 especifica los dos aspectos más importantes en la elección del inhalador por parte del médico:

1. Las preferencias del paciente. El médico debe de enseñar toda la gama de inhaladores con el objetivo que el enfermo pueda, en lo posible, elegir el que prefiera.

2. La comprobación del educador de que la técnica de inhalación es correcta por parte del enfermo. En este sentido, la educación sanitaria es fundamental para adiestrar inicialmente al paciente en la técnica inhalatoria y luego revisar de forma periódica la habilidad en el manejo del dispositivo, pues no es raro que los pacientes olviden con el paso del tiempo la técnica correcta ${ }^{8,11}$.

En el paciente, el inadecuado uso del dispositivo y la mala técnica en la maniobra de la inhalación pueden tener como consecuencia que el paciente no se adhiera al tratamiento; en un subanálisis del estudio TORCH, la escasa adherencia a la terapia inhalada en el grupo de pacientes con EPOC tratados con broncodilatadores, se asoció de forma significativa con un aumento de las hospitalizaciones y disminución de la supervivencia ${ }^{12}$.

Por otra parte, el conocimiento de los médicos sobre la terapia inhalada es muy deficiente ${ }^{13}$. En el estudio de Plaza y cols, 1.515 médicos de toda España que prescriben con frecuencia inhaladores implementaron un test de 11 preguntas de elección múltiple en el que se analizaban aspectos prácticos relacionados con el conocimiento de la terapia inhalada. Los resultados demostraron que sólo el 14,2\% de los médicos tenían un conocimiento adecuado de la misma. Además, el $72 \%$ identificó satisfactoriamente el principal paso en la 
técnica de los ICP y el $46,1 \%$ en la técnica de Ios IPS. El estudio puso de manifiesto dos deficiencias muy importantes relativas a la educación sanitaria de los pacientes en el uso de los dispositivos: sólo el 12,3\% de los médicos eligieron como característica más relevante a tener en cuenta a prescribir un dispositivo "las preferencias del paciente" y sólo el $27,7 \%$ respondieron que "siempre" verificaban la destreza del paciente en la utilización del dispositivo a la hora de la prescripción ${ }^{14}$. Parece indudable que deben de desarrollarse nuevos programas de formación y educación para mejorar el conocimiento de los dispositivos de inhalación y las técnicas de inhalación en los profesionales sanitarios que tratan a los pacientes con EPOC

\section{Conclusiones}

En la actualidad, de forma paralela al elevado número de fármacos disponibles en el tratamiento de la EPOC existe una amplia gama de dispositivos para su administración por vía inhalada. La elección adecuada del dispositivo y el conocimiento de la técnica inhalatoria es necesaria en el médico que atiende al enfermo, con el objetivo que su enseñanza activa se traduzca en el buen uso del dispositivo y la técnica para el paciente. Desafortunadamente, es muy común el manejo deficiente de los dispositivos y la técnica de inhalación en los pacientes, lo que genera un mal cumplimiento terapéutico y escasa adherencia que se traduce en un aumento en el número de hospitalizaciones y riesgo elevado de mortalidad. Por otra parte, el porcentaje de conocimiento global de la terapia inhalada no es mejor en los médicos y enfermeras, lo que dificulta que se realice un adecuado adiestramiento y educación sanitaria al paciente, necesarios en el deseado abordaje global en el tratamiento del paciente con EPOC. Con el objetivo de revertir esta tendencia, deben de desarrollarse nuevos programas de formación y educación en terapia inhalada a los profesionales sanitarios.

\section{Bibliografía}

1. GOLD. Global strategy for the diagnosis, management, and prevention of COPD, global initiative for chronic obstructive lung disease (GOLD) [Internet]. [updated 2014; Último acceso, 21 de febrero de 2014]. Disponible en: www.goldcopd.org

2. Guía de práctica clínica para el diagnostico y tratamiento de pacientes con Enfermedad Pulmonar Obstructiva Crónica (EPOC). Guía Española de la EPOC (GesEPOC). Grupo de trabajo de GesEPOC. Arch Bronconeumol. 2012; 48 (Supl 1): 2-58.

3. Cazzola M, Page CP, Calzetta L, Matera G. Pharmacology and therapeutics of broncodilatadors. Pharmacol Rev. 2012;64:450-504.

4. De Ávila Cabezón GR, Gonzálvez Rey J, Balaguer Balaguer EM. Las 4 reglas de la terapia inhalada. $1^{\mathrm{a}} \mathrm{E}$. 2015: pp 1-87.

5. Brau Tarrida A, Canela Pujol C, Murillo Anzano C. ¿Cómo se utilizan los dispositivos de inhalación?. FMC;2014;21:153-9.

6. Melani AS, Bonavia M, Clienti V, Cinti C, Lodi M, Martucci P, et al. Inhaler mishanding remains common in real life and is associated with reduced disease control. Respir Med. 2011;105:930-8.

7. Laube BL, Janssens HM, De Jongh FH, Devadason SG, Dhand R, Diot P, et al. European Respiratory Society; International Society for Aerosols in Medicine. What the pulmonary specialist should know about the new inhalation therapies. Eur Respir J.2011;37:1308-13.

8. Giner Donaire J, Tálamo Carrillo C, Plaza Moral V. Consenso SEPAR-ALAT sobre terapia inhalada. Arch Bronconeumol.2013:49 (Suppl 1): 2-14.

9. Yawn BP, Colice GL, Hodder R. Practical aspects of inhaler use in the management of chronic obstructive pulmonary disease in the primare care setting. International Journal of COPD. 2012;7:495-502.

10. Giner J. Torrejón M, Ramos A, Casan P, Granel C, Plaza V, et al. Preferencias de los pacientes en la elección de los dispositivos de inhalación en polvo. Arch Bronconeumol. 2004;40:106-9.

11. Burgos F. Terapia inhalada sin educación, un fracaso anunciado. Arch Bronconeumol.2002;38:297-9.

12. Vestbo J, Anderson JA, Calverley PM, Celli B, Ferguson GT, Jenkins C, et al. Adherence to inhaled therapy, mortality and hospital admission in COPD. Thorax.2009;64:939-43.

13. Plaza V, Calle M, Molina J, Quirce S, Sanchís J, Viejo JL, et al. Validación externa de las recomendaciones del Consenso Multidisciplinar sobre Terapia Inhalada. Arch Bronconeumol.2012;48:189-96.

14. Plaza V, Sanchis J, Roura P, Molina J, Calle M, Quirce S, et al. Physicians' knowledge of inhaler devices and inhalation techniques remains poor in Spain. J Aerosol Med Pulm Drug Deliv. 2012;25:16-22. 